\title{
Physiologische Untersuchungen an thermophilen Blaualgen
}

\author{
Von Erwin Bünning und Hedwig Herdtle \\ Aus den Botanischen Instituten der Universitäten Straßburg und Köln \\ (Z. Naturforschg. 1, 93-99 [1946]; eingegangen am 5. November 1945)
}

An mehreren thermophilen Blaualgen wurden im Freiland und im Laboratorium Beobachtungen vorgenommen. Genauer untersucht wurde Oscillatoria geminata.

Die Assimilation erreicht ihren Höchstwert bei etwa $40^{\circ}$, d. i. wenige Grad unterhalb der erträglichen Höchsttemperatur.

Die intensivste Atmung wird ebenfalls bei $40^{\circ}$ erreicht. Die Atmung steigt mit zunehmender Temperatur auffällig wenig. Im ganzen besteht eine für andere Pflanzen nicht bekannte Ähnlichkeit in der Temperaturabhängigkeit der Assimilation und Atmung. Hierdurch wird gewährleistet, daß auch bei hohen Temperaturen noch ein Assimilationsüberschuß erreicht werden kann.

Die Geschwindigkeit des Längenwachstums läßt das gleiche Temperaturoptimum von $40^{\circ}$ erkennen.

Die Temperaturkoeffizienten des Absterbeprozesses bei Temperaturen zwischen 50 und $70^{\circ}$ lassen keine erhebliche Abweichung von den für andere Pflanzen bekannten Werten erkennen.

Aus den Beobachtungen wird gefolgert, daß sich die thermophilen Blaualgen durch einen Plasmazustand auszeichnen, der etwa mit dem anderer Pflanzen in Phasen der Resistenzerhöhung vergleichbar ist. Dieser Zustand, vielleicht bedingt durch einen verminderten Gehalt an freiem Wasser, wirkt hemmend auf die Atmungsintensität, indem er die Geschwindigkeit der chemischen Reaktionen, etwa durch Diffusionshemmung, beeinträchtigt. Dieser Plasmazustand verursacht gleichzeitig eine erhöhte Stabilität. Die Stabilitätserhöhung, die auch die Möglichkeit einer Assimilation bei hoher Temperatur bedingt, ist auf Kosten einer Verminderung der Lebensintensität erreicht, so daß die thermophilen Blaualgen nur unter den für andere Arten ungünstigen extremen Bedingungen (hohe Temperatur, giftige Lösungen) iiberlegen sind, während ihr Wachstum sonst für die Konkurrenz zu schwach ist.

O bwohl zahlreiche Forscher dasVorkommen von Blaualgen in heißen Quellen noch bei Temperaturen von $60-70^{\circ}$ beobachtet haben (vergl. etwa Molisch ${ }^{1}$, der auch zahlreiche eigene Beobachtungen zusammengestellt hat), sind über die physiologischen Grundlagen der Anpassung an diese hohen Temperaturen bisher nur Vermutungen geäußert worden. So meint beispielsweise Molis ch, in den Organismen der heißen Quellen seien Eiweißarten, die erst bei besonders hoher Temperatur koagulieren. Molisch und andere Autoren denken dabei auch an einen Koagulationsschutz durch Stoffe, die dem Plasma beigemischt sind.

Durch Freilandbeobachtungen, die der eine von uns (B.) an Blaualgen in heißen Quellen Sumatras und Javas vornahm, wurde die vorliegende Untersuchung angeregt. Die Durchführung der experimentellen Arbeit lag zum größten Teil in den Händen von $H$. Herdtle.

\section{Beobachtungen im Freiland}

An etwa 15 verschiedenen Stellen wurden auf Sumatra und Java Erfahrungen über das Vorkom-

1 H. Molis ch, Pflanzenbiologie in Japan. Jena 1926. men von thermophilen Blaualgen gesammelt. Die höchste Wassertemperatur, bei der Blaualgen sich noch $\mathrm{zu}$ entwickeln vermochten, betrug $70^{\circ} \mathrm{C}$. Temperaturen von $70-75^{\circ}$ wurden von mehreren Arten ertragen, wenn sie nur wenige Stunden einwirkten. Die Artenzahl war bei Temperaturen bis etwa $50^{\circ}$ groß. Bei noch höheren Temperaturen wurden namentlich gefunden: Phormidium laminosum, Mastigocladus laminosus, einige Oscillatoria-, Lyngbya- und Anabaena-Arten.

Die für die Blaualgen erträgliche Höchsttemperatur war an den einzelnen Standorten ganz verschieden. In einigen Quellen wurden Cyanophyceen noch bei $70^{\circ}$ gefunden, in anderen fehlten sie schon bei $50^{\circ}$, obwohl das Wasser, sobald es sich auf Temperaturen zwischen $45-50^{\circ}$ abgekühlt hatte, eine üppige Blaualgenvegetation ermöglichte. Zur Erklärung dieser Verschiedenheiten könnte man vermuten, daß die noch bei besonders hohen Temperaturen lebensfähigen Arten nicht weit verbreitet sind. Jedoch konnten jene Verschiedenheiten auch in einem und demselben Quellgebiet beobachtet werden. Mehrere weitere Beobachtungen führten zur Annahme, daß Unterschieden in den 
Assimilationsbedingungen eine entscheidende Rolle zufällt. Wo Blaualgen noch bei Temperaturen zwischen 65 und $70^{\circ}$ wuchsen, betrug die $\mathrm{CO}_{2}$-Konzentration der Luft unmittelbar über dem Wasser zwischen 2 und $5 \%$. Das Wasser dürfte sehr reich an Kohlensäure gewesen sein. Außerdem waren solche Standorte durch sehr hohe Lichtintensität ausgezeichnet. An schattigen Stellen, zu denen nur $1 / 15$ oder weniger des vollen Sonnenlichtes gelangte, gediehen Blaualgen trotz hoher $\mathrm{CO}_{2}$-Konzentration nicht bei Temperaturen über $50-55^{\circ}$. Ebenso fehlten die Blaualgen an den sehr hellen Punkten, wenn die $\mathrm{CO}_{2}$-Konzentration nur gering war. Offenbar sind also nach diesen Freilandbeobachtungen für das Blaualgenwachstum bei extrem hohen Temperaturen günstigste Assimilationsbedingungen notwendig.

Noch eine andere Beobachtung ist erwähnenswert: In Schwefelquellen, und zwar an Standorten, wo die Luft so reich an Schwefeldioxyd war, daß jegliche andere Vegetation verhindert wurde, wuchsen immer noch reichlich Blaualgen, dabei traten aber an den kühleren Stellen (bei Temperaturen zwischen 20 und $35^{\circ}$ ) fast nur die gleichen Arten auf, die auch für die heißen Quellen charakteristisch sind. Eine Bestimmung war in den meisten Fällen nicht möglich, jedoch konnte jene Übereinstimmung der Arten einwandfrei festgestellt werden. $\mathrm{Zu}$ diesen sowohl gegen hohe Temperatur als auch gegen starke Schwefeldämpfe resistenten Arten gehörten z. B. Oscillatoria formosa, O. animalis, O. terebriformis. Diese Arten waren nicht etwa auf die Gegenwart von Schwefeldioxyd oder Schwefelwasserstoff angewiesen, in gewöhnlicher Nährlösung ließen sie sich (bei $28^{\circ}$ ) leicht kultivieren. Trotzdem konnten sie in der freien Natur fast nur in den Schwefelquellen oder bei extrem hoher Temperatur gefunden werden; in anderem Wasser wurden sie, wenn dort überhaupt Blaualgen vorherrschten, fast völlig von anderen Arten verdrängt. Gegen hohe Temperatur resistente Arten scheinen sich also oft auch durch eine hohe Giftresistenz auszuzeichnen.

\section{Kultur im Laboratorium}

Nach thermophilen Blaualgen für die physiologische Analyse wurde in den Gewächshäusern der Botanischen Gärten Straßburg/E. und Würzburg sowie in Erde, Baumrinde usw. von Straßburg und Boxberg/Baden gesucht. Proben dieser Herkünfte wurden in Erlenmeyerkolben mit Nähr- lösung gebracht. Die Nährlösung wurde durch 10-faches Verdünnen folgender Stammlösung gewonnen:

$1000 \mathrm{~g}$ aqua dest., $0,5 \mathrm{~g} \mathrm{Ca}\left(\mathrm{NO}_{3}\right)_{2}, 0,1 \mathrm{~g} \mathrm{MgSO}_{4}, 0,2 \mathrm{~g}$ $\mathrm{K}_{2} \mathrm{HPO}_{4}$, ein Tropfen einer 1-proz. $\mathrm{FeCl}_{3}$-Lösung.

Bei den Versuchen mit Oscillatoria geminata, über die ausführlicher zu berichten sein wird, wurden je Liter der fertigen Nährlösung $3 \mathrm{ccm}$ einer Erdabkochung hinzugefügt.

Soweit es sich nur darum handelte, thermophile Blaualgen aufzusuchen, diese also noch nicht unmittelbar für Stoffwechselversuche usw. benutzt wurden, kamen auch Abweichungen von der oben genannten Nährlösung zur Anwendung.

Die Kolben mit dem in die Nährlösungen übertragenen Material wurden in Brutschränke gestellt. Die Metalltür der Schränke blieb offen, so daß diese nur durch eine doppelwandige Glastür verschlossen waren und eine Beleuchtung der Kulturen möglich wurde. Die Kulturen erhielten in Straßburg künstliches Licht (300 Watt-NeoluxLampe in $30 \mathrm{~cm}$ Abstand von der Glastür), späterhin natürliches Licht in unmittelbarer Fensternähe. Die Temperatur wurde durch mehrere Thermometer innerhalb der Brutschränke kontrolliert; auch in einige der Kolben selber wurden Thermometer direkt in die Nährlösung eingeführt. Bei den Kulturen in Straßburg (Oscillatoria geminata) wurde die Temperatur nur während der Lichtperiode ( 8 bis $20 \mathrm{Uhr}$ ) hoch gehalten, in der Dunkelperiode jedoch die Heizung des Thermostaten ausgeschaltet, um eine bessere Entwicklung durch Verhinderung zu hoher Atmungsverluste zu erreichen. Bei den späteren Versuchen mit anderen Arten zeigte sich jedoch, daß die thermophilen Algen ebenso gut wachsen, wenn die Temperatur in der Dunkelperiode ebenso hoch ist wie in der Lichtperiode.

Aus dem Material von den Straßburger Gewächshäusern wurde eine Oscillatoria geminata isoliert, die sich bei den eingehender $\mathrm{zu}$ beschreibenden Assimilations- und Atmungsversuchen gut bewährte. Diese Art ertrug Temperaturen bis etwa $45^{\circ}$, war also nicht extrem thermophil. Aus den Würzburger Gewächshäusern konnte noch eine größere Zahl von Arten gewonnen werden, von denen mehrere auch bei noch höherer Temperatur gediehen:

Fischerella spec. Nach Geitler (Cyanophyceen, in Paschers Süßwasserflora) nicht bestimmbar. Bis etwa $55^{0}$ gut wachsend. 
Mastigocladus laminosus, bis etwa $55^{0}$ gut wachsend.

Nostoc muscorum, ebenfalls bis $55^{0}$ wachsend.

Oscillatoria anguina, acuminata, formosa und Boryana bis etwa $50^{\circ}$ wachsend.

Phormidium luridum bis etwa $45^{\circ}$, Phormidium ambiguum bis etwa $48^{\circ}$ wachsend.

\section{Methodik der Assimilations-und} Atmungsversuche

Für die Stoffwechselversuche wurde nur Oscillatoria geminata benutzt. Diese Art wächst in kleinen, zunächst lockeren blaugrünen Flöckchen, die frei in der Nährlösung schwimmen und sich bei starker Assimilation durch den entstehenden Sauerstoff an der Oberfläche ansammeln. Die Fäden sind nur von einer ganz schwachen Gallerthülle umgeben. Die Wachstumsweise dieser Art und die Beschaffenheit der Kolonien ermöglichte eine gute Verwendung für Stoffwechseluntersuchungen mit der W a rburg-Apparatur.

Kulturen, die kein normales und frisches Aussehen boten, z. B. solche, in denen die Zellen eine Vakuolisation zeigten, wurden für die Versuche nicht benutzt.

Die rechteckigen Assimilationskammern der Warburg-Apparatur wurden durch eine seitliche Öffnung mit der Algenflüssigkeit beschickt und die für die Assimilation benötigte Kohlensäure in einem Kaliumcarbonat-bicarbonat-Gemisch hinzugegeben. Ein Liter dieses Gemisches enthielt $8,52 \mathrm{~g} \mathrm{KHCO}_{3}$ und $2,072 \mathrm{~g} \mathrm{~K}_{2} \mathrm{CO}_{3}$ in $1000 \mathrm{ccm}$ aqua dest. Auf $7 \mathrm{ccm}$ Algenflüssigkeit kamen $2 \mathrm{~cm}$ des Carbonatgemisches in je ein Assimilationsgefäß. Zur $\mathrm{CO}_{2}$-Absorption dienten $0,3 \mathrm{ccm}$ konz. Kalilauge, die in den seitlichen Ansatz des Gefäßes gebracht wurden.

Bei den Assimilationsversuchen diente als Lichtquelle eine 75-Watt-Soffittenlampe, die sich im Thermostaten der Apparatur etwa $2 \mathrm{~cm}$ unter den Assimilationsgefäßen befand.

\section{Abhängigkeit der $\mathrm{CO}_{2}-\mathrm{Assimilation}$} von der $\mathrm{CO}_{2}-\mathrm{Konzentration} \mathrm{und} \mathrm{vonder}$

\section{Lichtintensität}

Auf eine Bestimmung der absoluten Assimilationsleistung einer bestimmten Menge des Algenmaterials mußte verzichtet werden, da Gewichtsbestimmungen der benutzten geringen Materialmengen nicht möglich waren. Die Assimilation unter den jeweils zu untersuchenden Bedingungen wurde daher (ebenso wie später die Atmung) im- mer mit der unter anderen, vorher gebotenen $\mathrm{Be}$ dingungen gefundenen verglichen. Für die Anwendung dieses Verfahrens mußte zunächst geprüft werden, ob die Assimilation der in die Apparatur übertragenen Proben über längere Zeit hinweg ausreichend konstant bleibt.

Versuche, in denen die Assimilation unter konstanten Bedingungen mehrere Stunden hindurch fortlaufend bestimmt wurde, zeigten, daß die Stoffwechselintensität während der für die Auswertung der späteren Versuche zu fordernden Zeiten ausreichend konstant bleibt. Nur in der Nähe der höchsterträglichen Temperaturen $\left(43-45^{\circ}\right)$ macht

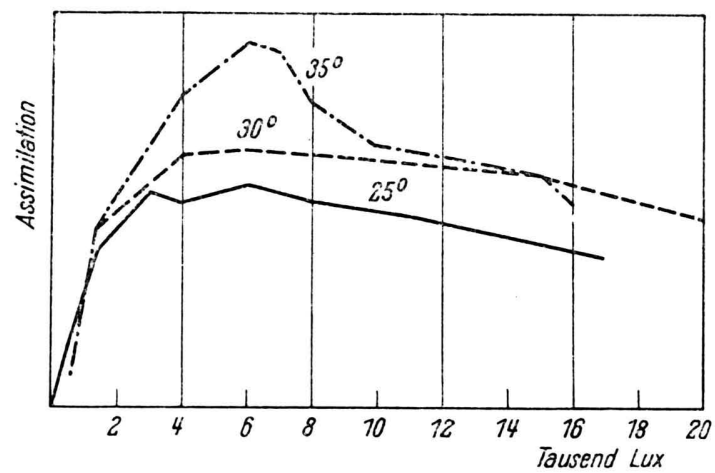

Abb. 1. Assimilation von Oscillatoria geminata bei verschiedenen Lichtintensitäten. Die drei Versuche wurden nicht mit gleichen Algenmengen durchgeführt, so daß die Kurven keinen Anhaltspunkt für die relative Assimilationsintensität bei verschiedenen Temperaturen geben; sie sollen nur zeigen, daß unabhängig von der Temperatur bei etwa 6000 Lux ein Assimilationsoptimum erreicht wird.

sich nach längerer Zeit im Abfall der Assimilationsintensität eine Schädigung bemerkbar.

Für die Hauptversuche mußte noch die optimale Lichtintensität und die optimale Kohlensäurekonzentration bestimmt werden. Der bei den eben genannten Versuchen erkennbare geringe Abfall der Assimilation nach längerer Zeit könnte auf einen Carbonatmangel hinweisen, jedoch zeigte sich, dafs bei Zugabe von 3 anstelle von $2 \mathrm{ccm}$ Carbonatgemisch die Assimilation noch rascher absinkt.

Die für die Ermittlung der optimalen Lichtintensität dienenden Versuche wurden bei verschiedenen Temperaturen (25,30 und $\left.35^{\circ}\right)$ ausgeführt (Abb.1). Als optimale Lichtintensität erwiesen sich überraschenderweise in allen drei Versuchsreihen schon 6000 Lux. Eine Temperaturabhängigkeit der Lage dieses Optimums, wie sie von anderen Autoren für andere Pflanzen beschrieben worden ist, 
wurde nicht gefunden. Man muß O.geminata demgemäß als eine typische, an schwaches Licht angepaßte Schattenpflanze bezeichnen. Das ist um so auffälliger, als sonst Schattenpflanzen mehr an niedrige Temperaturen angepaßt zu sein pflegen, und nach den einleitend genannten Freilandbeobachtungen auch bei den an extrem hohe Temperaturen angepaßten Blaualgen hohe Lichtintensitäten für die Lebensmöglichkeit bei diesen Temperaturen wichtig sind.

\section{Die Temperaturabhängigkeit der Assimilation}

Es wurde jeweils zunächst die wahre Assimilation bei $20^{\circ}$ bestimmt. Hierzu wurden erst bei Dun-

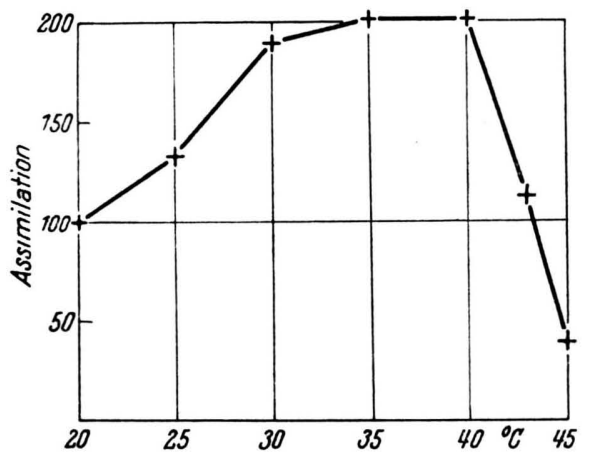

Abb. 2. Relative Assimilationsintensität von Oscillatoria geminata bei verschiedenen Temperaturen.

kelheit 3-4 Atmungsmessungen, sodann 3-4 Assimilationsmessungen und anschließend nochmals 3-4 Atmungsmessungen vorgenommen. Jede Einzelbestimmung dauerte 10 Min. Das Mittel aus allen Atmungsmessungen vor und nach den Assimilationsbestimmungen wurde zum Mittelwert der Assimilation gezählt und so die ,wahre Assimilation" bei $20^{\circ}$ errechnet. Für die Assimilationsmessung diente hier wie bei allen weiteren Versuchen die oben genannte optimale Intensität von $6000 \mathrm{Lux}$. Sodann wurde der Thermostat der Warburg Apparatur auf die gewünschte höhere Temperatur gebracht und in derselben Weise wiederum die wahre Assimilation ermittelt, die dann in Prozent der bei $20^{\circ}$ gefundenen ausgedrückt wurde.

Zur Kontrolle wurde schließlich noch einmal die wahre Assimilation bei $20^{\circ}$ ermittelt, um, besonders bei sehr hohen Temperaturen, eine etwaige Schädigung festzustellen. Die durchschnittliche Dauer eines solchen vollständigen Versuchs betrug etwa 8 Stunden.
Die Ergebnisse sind in Abb. 2 dargestellt. Die Assimilationsintensität steigt von $20-30^{\circ}$ rasch an, bleibt von $30-40^{\circ}$ in der gleichen Höhe und fällt zwischen 40 und $45^{\circ}$ rasch ab. Das Optimum ist also sehr breit und wenn man überhaupt einen höchsten Punkt annehmen will, so ist dieser bei etwa $40^{\circ} \mathrm{zu}$ suchen.

Es sei daran erinnert, daß dieses Optimum bei den an gewöhnliche Temperaturen angepaßten Pflanzen nach den Untersuchungen mehrerer Autoren bei Temperaturen zwischen 20 und $30^{\circ}$ erreicht wird. Vergleichbar ist das Verhalten unserer Oscillatoria in dieser Hinsicht mit demjenigen einiger höherer Pflanzen und Flechten aus tropischen Gebieten, die St ocker ${ }^{2}$ untersucht hat.

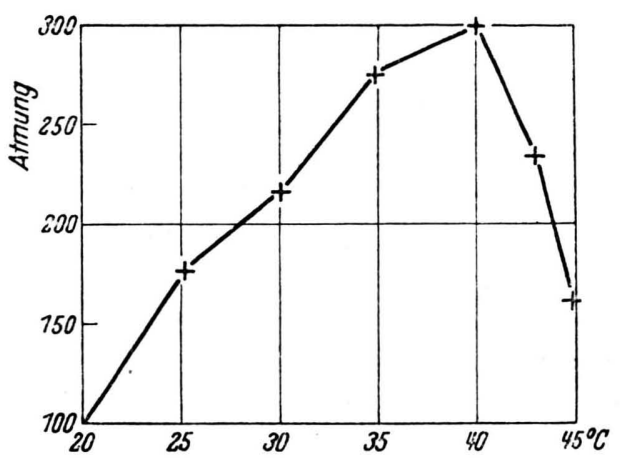

Abb. 3. Relative Atmungsintensität von Oscillatoria geminata bei verschiedenen Temperaturen.

\section{Die Temperaturabhängigkeit der}

\section{Atmung}

Die Werte für die Atmung bei verschiedenen Temperaturen wurden den oben besprochenen Assimilationsversuchen entnommen und in Abb. 3 zusammengestellt. Ebenso wie bei der Darstellung der Beziehung der Assimilation zur Temperatur in Abb. 2 ist auch hier wieder jeder Kurvenpunkt ein Mittelwert aus mehreren Versuchen, von denen jeder einzelne seinerseits 6 Einzelbestimmungen (nämlich 3 vor und 3 nach der Beleuchtung) zusammenfaßt.

Das Maximum der Atmung wird also ebenso wie das der Assimilation bei etwa $40^{\circ}$ erreicht, überhaupt zeigt die Temperaturabhängigkeit der Atmung eine auffällige Ähnlichkeit mit der der Assimilation. Am deutlichsten wird diese von den an gewöhnliche Temperaturen angepaßten Pflanzen

2 O. St ocker, Assimilation und Atmung westjavanischer Tropenbäume. Planta 24, 402 [1935]. 
nicht bekannte Ähnlichkeit, wenn wir die Temperaturkoeffizienten $\left(Q_{5}-\right.$ Werte $)$ zusammenstellen:

\begin{tabular}{c|c|c}
\hline Temperaturbereich & $Q_{5}$ der Assimilation & $Q_{5}$ der Atmung \\
\hline $20-25^{0}$ & 1,35 & 1,77 \\
$25-30^{0}$ & 1,41 & 1,22 \\
$30-35^{0}$ & 1,06 & 1,29 \\
$35-40^{\circ}$ & 1,00 & 1,09 \\
$40-45^{0}$ & 0,03 & 0,54
\end{tabular}

Daß unsere Alge noch bei relativ hoher Temperatur einen Assimilationsüberschuß erzielen kann, liegt also sowohl im fortgesetzten Assimilationsanstieg bis in den Bereich hoher Temperatur hinein als auch in der relativ geringen Steigerung der Atmung mit zunehmender Temperatur begründet.

\section{Assimilation und Atmung bei niedri- ger Temperaturkultivierter Algen}

Oscillatoria geminata konnte auch bei niedriger Temperatur, nämlich an einem Gewächshaus-Standort mit Extremtemperaturen von +15 und $+27^{\circ}$ kultiviert werden. Auch mit solchen Kulturen wurden Assimilations- und Atmungsmessungen vorgenommen. Auf die Ergebnisse braucht hier nicht im einzelnen eingegangen zu werden, da die Abweichungen gegenüber den vorher beschriebenen Versuchen nicht groß sind. Wiederum wurden Optima der Assimilation und der Atmung bei $40^{\circ}$ gefunden. Bemerkenswert ist jedoch, daß die Assimilation bei niedriger Temperatur $\left(20-30^{\circ}\right)$ relativ stärker ist als bei den in hoher Temperatur aufgezogenen Algen. Das gilt, jedoch in geringerem Maße, auch für die Atmung. Im ganzen sind also die Stoffwechselleistungen der bei niedriger Temperatur kultivierten Algen im Bereich dieser niedrigen Temperaturen etwas stärker als die Leistungen der bei hoher Temperatur kultivierten Algen im gleichen Bereich niedriger Temperatur, jedoch ist von dieser Förderung die Assimilation mehr betroffen als die Atmung, so daß die bei niedriger Temperatur kultivierten Algen im Bereich dieser niedrigen Temperaturen einen größeren Stoffgewinn erreichen als die anderen.

\section{Die Temperaturabhängigkeit des Längenwachstums}

Kleine Klümpchen der O.geminata wurden in einem Tropfen Wasser zwischen zwei Objektträgern zerrieben. Der erhaltene mit einzelnen Faden- stücken angefüllte Wassertropfen wurde in einer Petrischale auf Kieselgallerteboden möglichst gleichmäßig verteilt. So konnten einzelne Fäden im Mikroskop beobachtet und mit dem Zeichenapparat gezeichnet werden. Die Fäden waren an ihrer Form und ihrer Lage zueinander immer wieder leicht erkennbar, weil Kriechbewegungen nicht erfolgten.

Während der Versuche befanden sich die Petrischalen in einem Brutschrank, der um $90^{\circ}$ umgelegt worden war, damit eine Beleuchtung (mit 3000 Lux) von oben möglich wurde. Die Schalen wurden jeweils abends beimpft, am nächsten Morgen begannen dann die Messungen, zu denen bei der jeweils

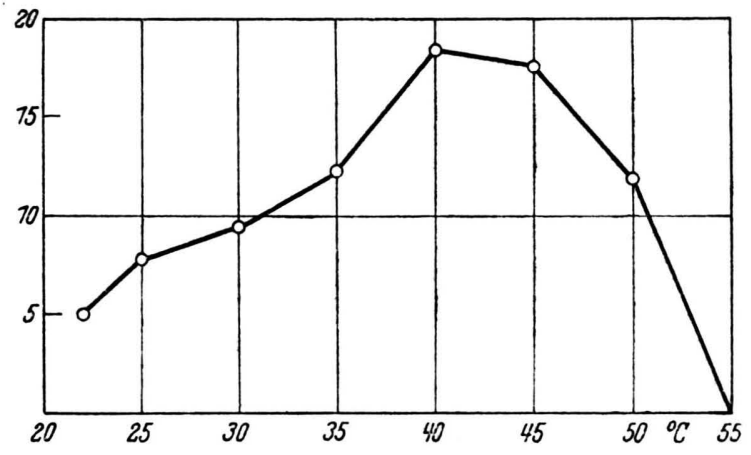

Abb. 4. Längenwachstum (Ordinate, in Prozent je Stunde) von Oscillatoria geminata bei verschiedenen Temperaturen.

zu prüfenden Temperatur der Zuwachs von je 10 bis 15 Algenfäden aus einer Schale in Abständen von einer Stunde durch Ausmessen der mit einem Zeichenapparat festgehaltenen Länge bestimmt wurde. Die Messungen wurden an jedem Faden durch mehrere Stunden hindurch fortgesetzt. Es ergaben sich ziemlich starke Schwankungen, so daß für jeden Endwert etwa 50 Messungen vorgenommen werden mußten.

Die Ergebnisse sind in Abb. 5 dargestellt. Die Beziehung zur Temperatur ist etwa so, wie man es nach dem Verlauf der Assimilationskurve erwarten konnte. Auffällig ist nur, daß das Maximum erst zwischen 50 und $55^{\circ}$ erreicht wird, jedoch ist dabei zu berücksichtigen, daß die Temperaturmessungen hier nicht so zuverlässig sind, wie bei den Siroffwechseluntersuchungen, bei denen durch die Schüttelvorrichtung für einen völligen Temperaturausgleich gesorgt werden konnte.

In den Dunkelperioden zeigten die Fäden nur eine ganz unbedeutende Längenzunahme. 


\section{Die Temperaturabhängigkeit des} Hitzetodes

Die Temperaturkoeffizienten des Hitzetodes sind bekanntlich sehr hoch und etwa mit den Temperaturkoeffizienten der Hitzekoagulation von Eiweißkörpern vergleichbar. Die in der Einleitung zitierte Vermutung, daß sich thermophile Blaualgen durch ein Eiweiß auszeichnen, das bei hoher Temperatur nicht so leicht koaguliert, ließ Versuche wünschenswert erscheinen, bei denen die Absterbegeschwindigkeit der Oscillatoria geminata in Wasser von verschiedener Temperatur ermittelt wurde.

$\mathrm{Zu}$ diesen Versuchen wurden kleine Algenklümpchen verschieden lange Zeit in Nährlösungen übertragen, deren Temperatur vorher auf die jeweils zu prüfende Höhe gebracht worden war. Die Algen wurden nach Beendigung dieser Behandlung auf Kieselgallerte übertragen, um so, ähnlich wie bei den vorher beschriebenen Wachstumsversuchen, feststellen zu können, ob die Fäden wachstumsfähig, d.h. lebend geblieben waren. Konnte auch nach 8 Tagen noch kein Wachstum festgestellt werden, so wurde die Probe als tot bezeichnet.

Folgende Mittelwerte für die Absterbegeschwindigkeit wurden gefunden:

\begin{tabular}{c|c}
\hline \hline $\begin{array}{c}\text { Temperatur } \\
{ }_{0} \mathrm{C}\end{array}$ & $\begin{array}{c}\text { Zeit his zum Eintritt } \\
\text { des Hitzetodes }\end{array}$ \\
\hline 50 & 65 Strn. \\
5. & 750 Sek. \\
55 & $114 \quad \%$ \\
60 & $2 \cdot 2 \%$ \\
65 & $4,6 \%$ \\
70 & $2,0 \%$
\end{tabular}

Danach errechnen sich beispielsweise folgende $Q_{10}$-Werte: Für den Bereich 55-65025 und für den Bereich $60-70^{\circ} 11$.

Diese Werte liegen etwa in der gleichen Höhe wie die von anderen Forschern bei mehreren an gewölınliche Temperaturen angepaßten Pflanzen gefundenen (vergl. etwa die Zusammenstellung bei Bělehrádek ${ }^{3}$.

Entsprechende Versuche wurden auch noch mit dem etwas höhere Temperaturen ertragenden $M a$ stigocladus laminosus ausgeführt. Für den Bereich 68 bis $75^{\circ}$ ergaben sich $Q_{10}$-Werte für die Geschwindigkeit des Absterbeprozesses zwischen etwa 15

3 J. Bělehrádek, Temperature and living matter. Berlin 1935. und 30, also wiederum keinerlei Besonderheit im Vergleich mit anderen Pflanzen.

Einige nur orientierende Versuche wurden auch mit Blaualgen aus den heißen Quellen Javas und Sumatras vorgenommen. Es handelte sich um eine nicht bestimmte Oscillatoria-Art, die bei $64^{\circ}$ in einer heißen Quelle wuchs. Bei $68^{\circ}$ waren zum Abtöten 5-10 Min. bei $75^{\circ} 10-20$ Sek. erforderlich.

Sowohl die Temperaturabhängigkeit der Absterbeprozesse bei hoher Temperatur als auch die absolute Höhe der Absterbegeschwindigkeit im Temperaturbereich von $50-75^{\circ}$ zeigen keinerlei Besonderheit gegenüber den bei anderen Pflanzen gefundenen Werten. Wenn wir beispielsweise fragen, wie hoch die Temperatur sein muß, um ein $\mathrm{Ab}$ sterben in 10-20 Sek. zu bedingen, so finden wir für die thermophilen Blaualgen zumeist Werte von $60-75^{\circ}$, gelegentlich vielleicht bis $80^{\circ}$ ( so ungefähr nach Beobachtungen auf Java und Sumatra bei den Formen, die noch bei $68-70^{\circ}$ entwicklungsfähig sind). Bei den an gewöhnliche Temperaturen angepaßten Pflanzen können wir die gleichen Temperaturen für eine Abtötung in jener Zeit finden (wobei natürlich auch Arten vorkommen, die erheblich hitzeempfindlicher sind).

Unsere Beobachtungen bieten also keinerlei Anhaltspunkt für die Annahme, daß sich das Plasma der thermophilen Blaualgen durch eine besondere Temperaturabhängigkeit der Koagulation seiner Bestandteile auszeichnet.

10. Die Ursachen für die Besonderheiten in der Temperaturabhängigkeit des St offwechsels

Die Stoffwechselversuche haben folgende Besonderheiten ergeben: Die Atmung steigt schon bei mittleren Temperaturen mit zunehmender Temperatur relativ wenig, die Atmungsintensität nimmt mit steigender Temperatur nicht so ungehemmt zu wie bei anderen, an gewöhnliche Temperaturen angepaßten Pflanzen, vielmehr läßt sich, schon bei niedrigen Temperaturen beginnend, ein fortgesetztes Absinken der Temperaturkoeffizienten feststellen. Andere Pflanzen zeigen dieses Absinken in einem solchen Ausmaß erst bei schädigenden Temperaturen, und zwar dann, wenn diese schädigenden Temperaturen längere Zeit einwirken. Die Assimilationsintensität steigt im Gegensatz zu dem von anderen Pflanzen bekannten Verhalten bis zur Nähe der erträglichen Höchsttemperatur. Im Gan- 
zen zeigt sich bei der untersuchten thermophilen Blaualge eine auffällige Ahnlichkeit in der Beziehung von Atmung und Assimilation zur Temperatur.

Wie lassen sich diese Besonderheiten erklären? Es ist wohl von vornherein nicht sehr wahrscheinlich, daß das Plasma der thermophilen Blaualgen chemisch eine wesentlich andere Konstitution besitzt als das anderer Blaualgen. Wie in anderen Fällen der Anpassung von Pflanzen an besondere Standortbedingungen dürfte es sich auch hier mehr um eine Frage des Plasmazustandes handeln, und dafür sprechen auch die Beobachtungen selber. Wenn die Atmung bei anderen Pflanzen immer mehr bis zur Erreichung der höchsterträglichen Temperatur ansteigt, so ist das ein Ausdruck des ungehemmten, durch eine Schädigung bedingten Freilaufs der Enzyme (der auch sehr bald zum raschen Absinken des Stoffwechsels führt). Bei den thermophilen Blaualgen fehlt dieser Enzymfreilauf; die niedrigen Temperaturkoeffizienten legen die Schlußfolgerung nahe, daß zum mindesten bei den höheren Temperaturen nicht mehr die chemischen Prozesse selber, sondern die für sie notwendigen Stoffdiffusionen auf die Reaktionsgeschwindigkeit begrenzend wirken. Anscheinend ist also die Plasmastruktur derart, daß die Diffusion der reagierenden Stoffe oder der Reaktionsprodukte gehemmt wird. Wenn wir die neueren Untersuchungen über die Ursachen herabgesetzter Intensität der Lebensprozesse in Pflanzenzellen berücksichtigen, so ist als weitere Ursache jener Besonderheit vor allem an den Wassergehalt zu denken. Eine Verminderung der Menge freien Wassers bedingt sowohl eine Herabsetzung der Geschwindigkeit biochemischer Reaktionen als auch zugleich eine Erhöhung der Resistenz, beispielsweise eine Erhöhung der Resistenz gegen hohe Temperaturen. Die Plasmakolloide werden in einem solchen Zustand der Verminderung der Menge freien Wassers zugunsten des durch elektrische Kräfte fest an die Kolloide gebundenen stabiler gegen alle sonst schädigenden Einflüsse wie Gifte, Hitze usw. In diesem Zusammenhang sei an die schon vorher erwähnte Beobachtung erinnert, daß sich viele thermophile Blaualgen auch durch eine hohe Giftresistenz auszeichnen.

Die höhere Stabilität der Plasmakolloide kann auch den Assimilations- und Wachstumsverlauf erklären. Je stabiler die Kolloide sind, um so höher liegt die Temperatur, bei der sich die Schädigung der für die Syntheseleistungen erforderlichen Plasmastruktur bemerkbar machen kann.

Nun ist aber zu berücksichtigen, daß eine durch Verminderung des Gehalts an freiem Wasser bedingte Stabilitätserhöhung der Kolloide, die wir aus dem Stoffwechselverhalten erschließen, nicht nur mit einer erhöhten Resistenz, sond ${ }^{\circ}$ n auch mit einer verminderten Intensität der Lebrnsleistungen verknüpft sein muß. D. h. wenn sich die Anpassung der thermophilen Blaualgen an ihre Lebensbedingungen durch Besonderheiten des Plasmazustandes erklärt, die auch bei anderen Pflanzen im Zusammenhang mit Resistenzerhöhungen vorkommen, so muß, ebenso wie bei diesen im Stadium der Resistenzerhöhung, eine allgemeine Verminderung der Aktivität gegeben sein.

Die geringere Lebensintensität der thermophilen Blaualgen kann tatsächlich, vor allem in der Wachstumsintensität, beol,achtet werden. Wurden die Algen mit anderen, nur bei gewöhnlicher Temperatur wachsenden, gemeinsam in Kolben geimpft und bei Temperaturen von $30-35^{\circ}$ in den Brutschrank gestellt, so entwickelten sich die nichtthermophilen stets schneller als die thermophilen, die oft sogar ganz unterdrückt wurden, obwohl jene Temperatur mehr dem Optimum der thermophilen als dem der nichtthermophilen genähert war. Besonders auffällig langsam war das Wachstum der noch über $45^{\circ}$ entwicklungsfähigen Arten, wie z. B. Mastigocladus. Es dauerte 4-6 Wochen, bis sich von dieserArt nach derImpfung makroskopisch leicht sichtbare Kulturen entwickelt hatten, und zwar auch dann, wenn die Kultur nicht bei der höchsterträglichen Temperatur, sondern bei $30-45^{\circ}$ erfolgte.

Die Arten, die auf Java und Sumatra in heißen Quellen und in Schwefelquellen niedriger Temperatur vorherrschen, werden wenige Meter weiter entf $\rho$ rnt in weniger giftigem und weniger heißem Wasser von anderen Arten verdrängt, obwohl die gift- und hitzeresistenten Formen im Laboratorium in gewöhnlicher Nährlösung bei mäßiger Temperatur $\left(28^{\circ}\right)$ ebenso gut oder besser gedeihen als in Schweflige Säure und Schwefelwasserstoff enthaltenden Lösungen.

Endlich sei noch erwähnt, daß nach Freilandbeobachtungen auf Java und Sumatra die Neubesiedlung an Steinen usw., die in heiße Quellen gelegt wurden, auch an den Stellen auffällig langsam erfolgte, wo die Temperatur nur $50-55^{\circ}$ betrug, obwohl reichlich Oscillatoria vorhanden war, die bis zu $68^{\circ}$ ertrug. 